\title{
NAVIGATION AMONG OBSTACLES BY THE COOPERATION OF TRINOCULAR STEREO VISION SYSTEM AND LASER RANGEFINDER
}

\author{
J. R. Asensio J. M. M. Montiel L. Montano \\ Dep. de Informática e Ingeniería de Sistemas \\ Universidad de Zaragoza \\ María de Luna, 3, E-50015 Zaragoza, SPAIN \\ URL: http://www.cps.unizar.es/deps/DIIS/robot/
}

\begin{abstract}
In this paper a technique for robot navigation based on the cooperation of two complementary sensors is presented. The trajectory is planned obtaining the next location to be reached by means of a stereo vision system. The motion towards this location is carried out using a reactive obstacle avoidance navigation technique in unstructured environments without a priori map. The information about the environment is provided to the robot by two complementary sensors: a trinocular vision system and a laser rangefinder. The laser sensor is used for obstacle avoidance. Experimental results are reported, which show the robustness of the proposed techniques.
\end{abstract}

Keywords: Mobile Robots, Robot Navigation, Obstacle Avoidance, Computer Vision, Stereo Vision, Range finders, Real time

\section{INTRODUCTION}

Several kinds of sensors are used to acquire information from the environment to carry out robot navigation with obstacle avoidance. Multiple ultrasonic sensors, 2D or 3D laser rangefinder data sensors, vision systems and combinations of them are used. Up to now, most of proposed methods are based on a priori information, which is integrated in a grid map where location of walls, known obstacles and free ways are reflected. With respect to reactive navigation techniques, several works have proposed methods using artificial potential fields (Khatib, 1986), (Borenstein and Koren, 1989), and (Ratering and Gini, 1993).

Stereovision using straight segments are well adapted to reconstruct polyhedral scenes. Polyhedral objects are common in man made environ- ments. Trinocular stereo has been used to reconstruct the environment for mobile robot navigation in indoor environments (Ayache, 1991),(Zhang and Faugeras, 1992). Straight segment features allow to apply the previous knowledge that a door is a polyhedral object. The usage of a third camera (respect the usage of only two) allows the reconstruction of the segments aligned with the epipolar line of a camera pair; besides the robustness of the matches are improved.

In (Gilg and Schmidt, 1994) a navigation system based on straight segments detected by a unique camera is proposed. They report the ability of the straight segments to represent landmarks for intermediate navigation goals. They use the intersection between the walls and the floor as feature; they also reported weakness of the obstacle avoidance using only straight segments, suggesting the usage of laser sensors.

1 This work was partially supported by spanish CICYT projects TAP94-0390 and TAP97-0992-C02-01 
In our approximation, trinocular vision instead of monocular allows to extract full 3D information from the images, improving the robustness of the vision information. However, stereo features only produces a wire-frame model of the environment, unreliable information for obstacle avoidance. The usage of the laser sensor for navigation increases the performance of the low level navigation task.

In section 2 we present the robot platform. The trinocular vision system and the technique to reconstruct a $3 \mathrm{D}$ scene is briefly presented in section 3 . The proposed techniques to obtain the next location to be reached and to move avoiding the obstacles are explained in section 4 . There are two main tasks that must be achieved: locating the door, and navigating among obstacles. In section 5 we present experimental results in which we show how cooperation between the two sensors is made. Some conclusions are related in section 6 .

\section{THE ROBOT AND ITS SENSORIAL SYSTEM}

The Labmate robot is a differentially driven mobile robot (developed by Helpmate Robotics, Inc). It has two active and four passive wheels. Its maximum speed is $1 \mathrm{~m} / \mathrm{sec}$. The on-board software allows a host computer to control the robot. A control software has been developed to have a virtual controller from a host computer.

Stereo trinocular system is composed of $3 \mathrm{CCD}$ monocrome cameras connected to each of the RGB inputs of a color frame grabber, so the three images are taken synchronizedly. The 3D lidar laser rangefinder (TRC), radially scans the environment around the robot. The maximum range is 6.5 meters, and the accuracy of the distance measurement is $2.5 \mathrm{~cm}$. irrespective of the distance to the target. The location of trinocular vision system and 3D laser sensor on the robot structure can be viewed in Fig. 1.

\section{THE TRINOCULAR VISION SYSTEM}

The stereovision system is feature based one. The geometrical feature used is the straight segment, so the final solution reported by the trinocular system is a wire-frame reconstruction. Indoor scenes can be accurately represented with straight segments because most of the objects in the scene are man made. The matching of the segments in the three images considers both the collinearity and the overlapping. Collinearity considers that the supporting lines of the matched segments must be collinear. Overlapping considers that the matched image segments corresponds to the same part of

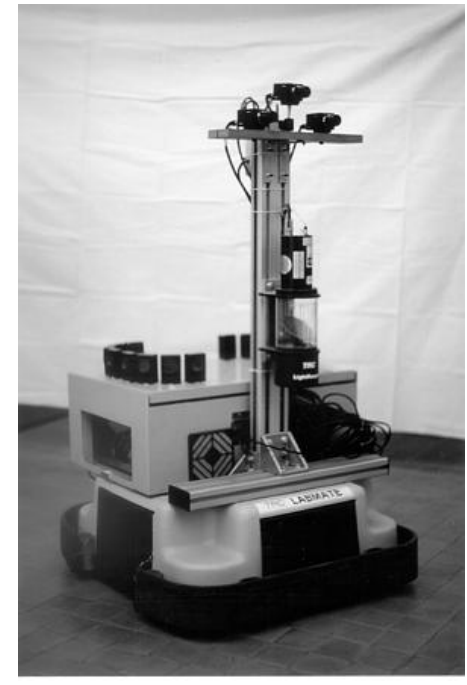

Fig. 1. Location of laser and trinocular vision systems on our Labmate robot.

the 3D supporting line. This condition is coded enforcing the matching between image segment midpoints. As collinearity is more reliable than overlapping, the first constraint in considered with a higher weight than the second.

The use of a third camera has two advantages. On one hand, matching is more robust because most of the spurious matches with only two images are rejected when the third camera is considered. On the other hand, image segments aligned with the epipolar line of a camera pair cannot be reconstructed. The use of a third camera allows to determine the 3D location for segments in every orientation because at most a segment is parallel to one of the epipolar lines, but can be recovered considering the other camera.

The trinocular system models the uncertain geometrical information using a probabilistic model in all the stages of the process. The model used is the SPmodel (Tardós, 1992). So, the decision about the acceptance or rejection of a hypothesis is done with a $\chi^{2}$ test; because of this the threshold tuning is done from a physical basis such as image noise, camera location error and false negative probability $(\alpha)$ for the $\chi^{2}$ test. The wireframe reconstruction also includes a covariance matrix for representing the 3D segment location error. This matrix codes the location error in each coordinate of the location estimate and is used to test geometrical constraints in the door localization stage.

The system is able to match the segments with a low spurious rate, one or two spurious per reconstruction. This low rate is due to the usage of a probabilistic model and to the usage of the overlapping constraint. More details about the system can be found in (Martínez et al., 1995). 
The cameras has been calibrated using the Tsai calibration method (Tsai, 1987), using a mobile planar pattern. The image segments has been extracted using the method proposed by Burns in (Burns et al., 1986). The cameras form a triangle whose base and height are 310 and 80 $\mathrm{mm}$. respectively, because of that the precision for vertical segments are bigger than the precision for horizontal ones.

\section{DOOR LOCALIZATION AND NAVIGATION TECHNIQUES}

Our goal is robot's exiting a room. For this purpose, first the robot must locate the door. Once this task has been accomplished, it must go to that door while avoiding obstacles. As follows, cooperation between two kinds of complementary sensors - trinocular vision system and laser rangefinderis crucial to solve the proposed problem.

Recognizing and locating the door - that implies locating the next point of the trajectoryis achieved by trinocular system, because laser sensor can be unable to see all the room, due to its limited range. Moreover, obstacle avoidance is better performed by the laser sensor, because it obtains fast multidirectional 3D information about the environment as the laser rotates. Trinocular vision can detect 3D information about the object's edges, and basically builds a wireframe reconstruction of the environment. Hence, it makes difficult to detect the free space areas. Once again, limitations of a sensor are overcome by the other, solving the overall problem through the cooperation between two complementary sensors.

There is another situation when cooperation between both sensors arises: the trinocular system can detect if the door is open or closed when it is far from it, and the laser rangefinder sensor can verify it and obtain with more precision its location when the robot is near the door.

\subsection{LOCATING THE DOOR}

The doors in the room are recognized and located by means of a set of three or four 3D straight segments that can be candidates to be a door: a pair of vertical segments (lateral limits of the door), an horizontal segment over the vertical ones (top edge of the door), and sometimes the horizontal lower edge of the door. The horizontal lower edge is on the floor, and can be considered as a clue for a closed door.

The basic process to find the doors in the room is as follows. First, the three images from the trinocular system are taken. Using the method explained in the section 3 , the vertical segments that verify some constraints related to a possible door (maximum and minimum length) are obtained in each image. The trinocular technique is applied to match the segments in the three images and to compute the 3D localization of the matched segments. A test of length and a test of verticality is applied to the segments to choose only the segments that may belong to a door. The uncertainty of the location of the segments provided by the trinocular process is used in a $\chi^{2}$ test for the those properties. Only the pairs of the segments whose distance are bigger than a given minimum distance are selected as candidates to be a door. For each pair of segments, an horizontal segment is looked for at the top of the pair in the three images. Only the sets of three segments which verify segment perpendicularity condition and distance conditions between extreme points of the vertical and horizontal ones, are selected to be doors, using also a $\chi^{2}$ test based on the location uncertainty of the involved segments. Afterwards, the system tests if the door is open or closed, by looking for an horizontal segment in the bottom of the door. Among the doors selected, several criteria can be established to choose one door, for example, we choose the nearest door to the robot.

Obviously, the robustness of the proposed method depends on multiple factors. Illumination conditions are very important to have contrasted segments in the images. As the location uncertainty of the horizontal segments is bigger than the vertical segments, several parameters in the method must be tuned to take into account this fact when the perpendicularity test between segments and distance test between extreme points are computed.

Stereo vision detects the door location up to some uncertainty. Small uncertainty can be achieved in the direction where the door center is, due to the angular resolution of a CCD camera. However, depth uncertainty is bigger due to the limited cameras baseline. In any case, to navigate towards the door the system only need to know with some precision its localization in a direction approximately normal to the robot motion, no matter the precision in the direction of the robot motion. The navigation system based on the laser information must assure that the robot cross the door, correcting the accumulated error due to the odometry drift.

\subsection{REAL TIME OBSTACLE AVOIDANCE}

The proposed technique to navigate in a partially known environment is based on artificial potential field techniques. The robot moves in a field of forces: it is subjected to an attractive force from the goal, and to the resultant of repulsive forces 
from the walls and obstacles found during the motion. The 3D laser sensor provides information about obstacles located not only in front of the robot, but also at both sides.

The forces are computed as follows:

- Attractive force:

$$
\begin{aligned}
& F_{a}=\left\{\begin{array}{l}
k_{1} \quad \text { if } d \geq d_{\text {goal }} \\
\frac{k_{1}}{d_{\text {goal }}} d \text { otherwise }
\end{array}\right. \\
& \theta_{a}=\operatorname{atan} 2\left(y_{\text {goal }}-y_{\text {robot }}, x_{\text {goal }}-x_{\text {robot }}\right)
\end{aligned}
$$

where $d$ is the distance to the goal and $d_{\text {goal }}$ is a fixed distance to slowly stop the robot.

- Repulsive force for one point belonging to an obstacle:

$$
\begin{aligned}
& F_{r}= \begin{cases}k_{2}\left(\frac{1}{d}-\frac{1}{d_{\max }}\right)^{2} & \text { if } d \leq d_{\max } \\
0 & \text { otherwise }\end{cases} \\
& \theta_{r}=\pi+\operatorname{atan} 2\left(y_{\text {obst }}-y_{\text {robot }}, x_{\text {obst }}-x_{\text {robot }}\right)
\end{aligned}
$$

where $d$ is the distance to a point of the obstacle, and $d_{\max }$ is the maximum distance which generates repulsive force.

The robot environment is angularly sectored, and for each sector the nearest sensed point is selected. The selected point is used to represent the whole sector. So, the repulsive force for the sector is computed as the repulsive force for the unique selected point, applying Eqs. 2.

All the forces acting on the robot are considered to be applied on one point located at the middle of the robot front. We model the system as a real mobile robot and thus, the robot motion is computed from a robot dynamic model (Montano and Asensio, 1997). We define the state vector $\mathbf{x}=\left(\psi, \dot{\psi}, \dot{\varphi}_{r}\right)^{T}$ where $\psi$ is the robot orientation angle and $\dot{\varphi}_{r}$ is the angular speed of the right wheel. Thus, the state-space equation to compute the motion is:

$$
\dot{\mathbf{x}}=\mathbf{A} \mathbf{x}+\mathbf{B} \mathbf{u}
$$

where the components of $\mathbf{A}$ and $\mathbf{B}$ include physical parameters of the robot, and where the input vector $\mathbf{u}=(F \sin \theta, F \cos \theta)^{T}$ is computed by the controller from the resultant force $\mathbf{F}$ applied on the frontal middle point. More details about this model and the implementation of the controller can be found in (Montano and Asensio, 1997).

Using this equation to obtain the motion from the force $\mathbf{F}$, allows filtering the sudden changes in the force module and orientation, that can appear when the potential field method is used to drive the robot in an environment with unknown or mobile obstacles. Thus, its use provides smooth motions compatible with the robot mechanical constraints.
Laser information is updated each $100 \mathrm{~ms}$. with the arrival of data belonging to a lap. In order to compute the repulsive resultant force, the system integrates the 10 last scans (corresponding to a complete sweep) taking into account the robot moves, with the following method:

a) Correction in one lap. In each lap, the location of the sensed points is corrected to refer all the points to the same time, the final time of the lap.

As the system knows the lap time $t_{l}$, the sensor location in a previous time $t_{i}$ can be computed from the location at $t_{l}$, inside the same sample period:

$$
\begin{aligned}
& \left(\begin{array}{c}
x_{l}^{L} \\
y_{l}^{L} \\
\psi_{l}^{L}
\end{array}\right)=\left(\begin{array}{c}
x_{k}^{L} \\
y_{k}^{L} \\
\psi_{k}^{L}
\end{array}\right)+\Delta t_{l}\left(\begin{array}{c}
v_{k} \cos \psi_{k} \\
v_{k} \sin \psi_{k} \\
\dot{\psi}_{k}
\end{array}\right) \\
& \left(\begin{array}{c}
x_{i}^{L} \\
y_{i}^{L} \\
\psi_{i}^{L}
\end{array}\right)=\left(\begin{array}{c}
x_{l}^{L} \\
y_{l}^{L} \\
\psi_{l}^{L}
\end{array}\right)-\Delta t_{i}\left(\begin{array}{c}
v_{k} \cos \psi_{i} \\
v_{k} \sin \psi_{i} \\
\dot{\psi}_{i}
\end{array}\right)
\end{aligned}
$$

where $v_{k}=\dot{\varphi}_{r, k} r-\dot{\psi}_{k} d, \Delta t_{i}=t_{l}-t_{i}, \Delta t_{l}=t_{l}-t_{k}$. One point $p_{i}$ is associated to each sector, and a new location relative to the robot is computed for each point.

b) Integration of points from several laps. Each sample period, the sensorial system of the robot integrates the selected points in each laser lap and transforms their relative location to the current location of the sensor.

\section{EXPERIMENTAL RESULTS}

The main goal of this paper is to experimentally test the performance of the cooperation between a trinocular stereo system and a laser rangefinder based reactive navigation system to carry out a task without a priori map.

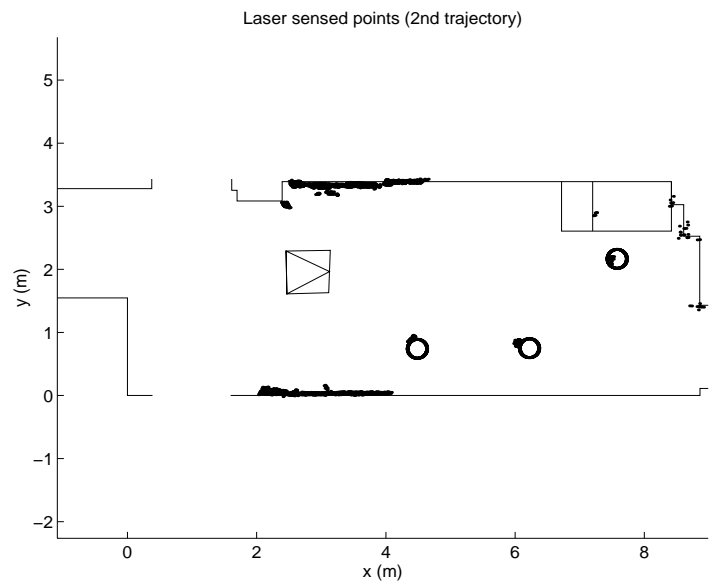

Fig. 3. Initial location of the robot in the second case, and set of points sensed by the laser at that location. 


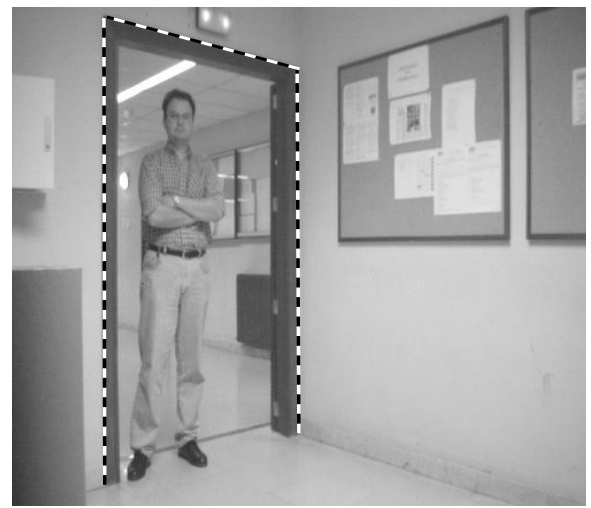

(a)

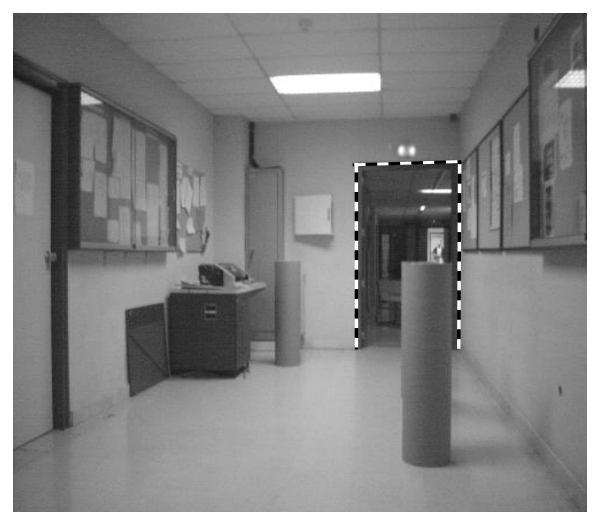

(c)

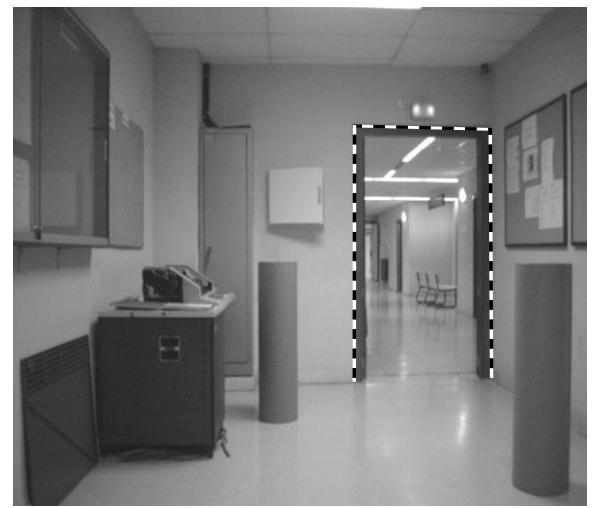

(b)

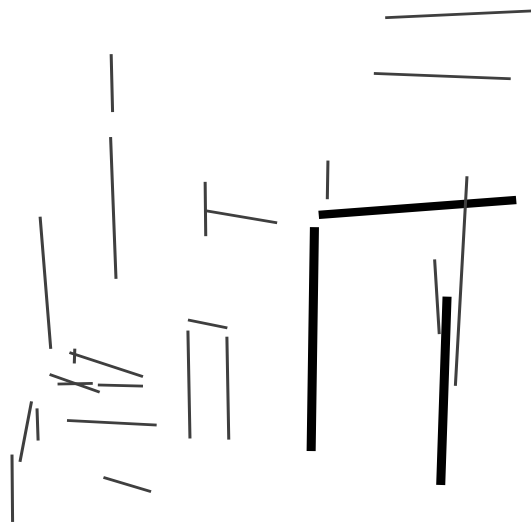

(d)

Fig. 2. (a) (b) and (c) are the first image in each case. (d) 3D Reconstruction of the scene for the Fig.

(b). The segments belonging to the door are marked with a thick line

The environment is a typical room, in which there are several doors and several obstacles that the robot must avoid as it moves. The system do not know where are neither the doors nor the obstacles and the robot must exit to the next room. In the experiment the robot vision system locates one door from some locations in the room. In Fig. 3 the information provided by the laser sensor is shown for a robot initial location. It can be seen that with this information is not possible to find any door in the room. Then, the vision system takes images and processes them to reconstruct the 3D scene and find a feature which match a door as defined in the model. In Figs. 2(a), (b), (c) the images taken by the left camera in three different initial locations in the room and the located doors are shown. In Fig. 2(d) the 3D reconstruction of the scene at one of the robot initial locations is represented. It is a complex scene with multiple segments detected, including those belonging to the obstacles. It can be also noted that the limits of the door are not seen as perpendicular segments in all the images, but the system is able to recognize the door in all the test cases.
When the system has located the door, the navigation system must drive the robot towards it, avoiding the obstacles in its way using the reactive navigation technique explained in 4.2. In Fig. 4 the trajectories followed by the robot in the three test cases are shown and the points used to compute the goal from the detected door are represented. For the motion control system, the points marked on the right of the door are the goals to be reached in each case.

It must be noted that the trajectories represented are not the real trajectories of the robot but the trajectories provided by the odometry. As there is an accumulated error in the robot location, the trajectories are displaced, and then it may seem that the robot bumps against the door. In spite of this location error, the navigation system allows to reach successfully the goal. In some cases, this problem can produce that the goal location computed relative to the robot location is near an obstacle, and the robot may be locked.

As a result of the experiment, we can conclude that the technique to locate doors is quite robust against different points of view of the vision system, that can recognize a feature such a door in 


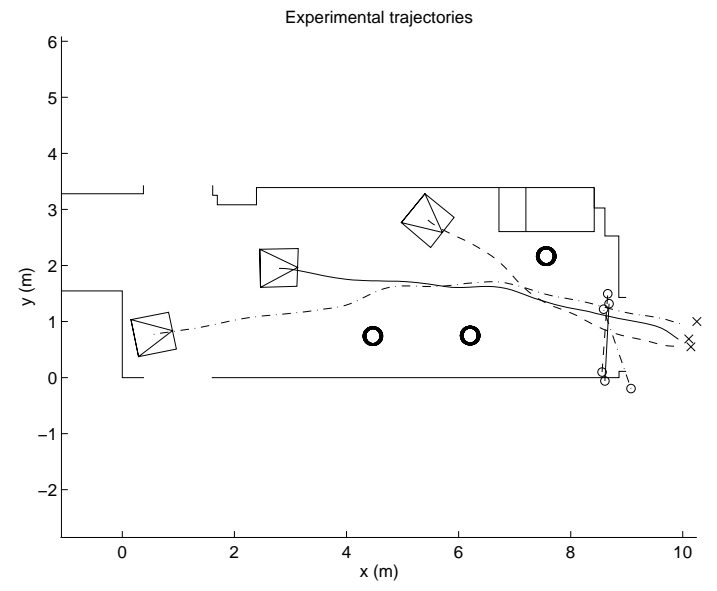

Fig. 4. Trajectories followed by the robot in the the three test cases .

a complex scene, even when there are obstacles or people in the scene. The navigation technique implemented allows to reach the goal avoiding the obstacles, showing as the cooperation between complementary sensors is necessary to accomplish such navigation task.

\section{CONCLUSIONS}

In this work we have shown how cooperation between two complementary sensors can be carried out in a navigation task, in which are not a priori information about the layout of the scene. We have presented a technique to recognize a particular complex feature in an indoor scene, such a door, by means a stereo vision system. This technique is robust in different robot locations. We have also shown that a reactive navigation technique using a laser rangefinder sensor is also robust in a partially cluttered environment, and that the complementary information from both sensors allows carry out successfully the task.

From the experimental results, some drawbacks has been stated. Since there are an accumulated error in the location provided by the odometry, the computed goal is displaced with respect to its real location. It can be corrected by using the vision and the laser information to relocate periodically the robot. The same information can be used to relocate the goal respect to the robot, when it is near, assuring that the robot reaches the goal with precision. The integration of navigation, map building and robot selflocation will be presented in future works. A more robust and efficient technique to find special features in the scene (doors, intersections in corridors, etc) is also pointed as future work.

\section{REFERENCES}

Ayache, N. (1991). Artificial Vision for Mobile Robots: Stereo Vision and Multisensory Perception. MIT Press, Cambridge, MA.

Borenstein, J. and Y. Koren (1989). Real-time obstacle avoidance for fast mobile robots. In: IEEE Transactions System, Man and Cybernetics. Vol. 19. pp. 1179-1187.

Burns, J.B., A.R. Hanson and E.M. Riseman (1986). Extracting straight lines. IEEE Trans. on Pattern Analysis and Machine Intelligence 8(4), 425-455.

Gilg, A. and G. Schmidt (1994). Landmarkoriented visual navigation of a mobile robot. IEEE Transactions on Industrial Electronics 41(4), 392-397.

Khatib, O. (1986). Real-time obstacle avoidance for manipulators and mobile robots. International Journal of Robotics Research 5, 90-98.

Martínez, J.M, Z. Zhang and L. Montano (1995). Segment-based structure from an imprecisely located moving camera. In: IEEE Int. Symposium on Computer Vision. Florida. pp. 182187.

Montano, L. and J. Asensio (1997). Real-time robot navigation in unstructured environments using a $3 \mathrm{~d}$ laser rangefinder. In: IEEERSJ International Conference on Intelligent Robots and Systems. Vol. 3. Grenoble, France. pp. 526-532.

Ratering, S. and M. Gini (1993). Robot navigation in a know environment with unknown moving obstacles. In: IEEE Int. Conference on Robotics and Automation. Vol. 3. Atlanta, Georgia. pp. 25-30.

Tardós, J.D. (1992). Representing partial and uncertain sensorial information using the theory of symmetries. In: IEEE Int. Conf. on Robotics and Automation. Nice, France. pp. 1799-1804.

Tsai, R.Y. (1987). A versatile camera calibration technique for high accuaracy $3 \mathrm{~d}$ machine vision metrology using Off-the-Shelf tv cameras and lenses. IEEE Journal of Robotics and Automation RA-3(4), 323-344.

Zhang, Z. and O. Faugeras (1992). A 3d world model builder with a mobile robot. Int. Journal of Robotics Research 11(4), 269-284. 